\title{
A connection between discrete individual-based and continuous population-based models: A forest modelling case study
}

\author{
Pablo Gómez-Mourelo, Marta Ginovart \\ Departament of Applied Mathematics, E.T.S. Ingenieros Industriales, Universidad Politécnica de Madrid, Spain \\ Correspondence: Marta Ginovart. Address: Department of Applied Mathematics III, Universitat Politècnica de Catalunya, \\ Campus Baix Llobregat, Esteve Terradas 8, 08860 Castelldefels (Barcelona), Spain. Telephone: 34-935-521-133. Email: \\ marta.ginovart@upc.edu.
}

Received: July 2, 2012

Accepted: September 13, 2012

Online Published: February 18, 2013

DOI : 10.5430/air.v2n2p28

URL: http://dx.doi.org/10.5430/air.v2n2p28

\section{Abstract}

Modelling is perceived as a way of dealing with real life activities. The aim of this work is to compare two approaches to study forest dynamics, namely discrete individual-based and continuous population-based models, in order to contribute to an improvement in their use among researchers. An analysis of the strengths, weaknesses, opportunities and threats of the two different approaches, jointly with a mention of the state-of-the-art, allows us to illustrate this discussion. We will also provide a bridge or connection between these two modelling methodologies. This link will be developed in detail in a particular study case. Firstly, an individual-tree based model to deal with dynamics of forests is presented. Secondly, this model is scaled up to a system of partial differential equations, which represents the limiting behaviour of the individualbased model.

\section{Key words}

Individual-based model, Population-based model, Discrete versus continuous model, Forest dynamics, Tree population

\section{I ntroduction}

To represent, analyse and discuss aspects or ideas related to biological systems from a general perspective modelling is a necessary tool. With this in mind, teaching science and doing research based on the elaboration of models is widely accepted in the academic community.

The discussion among theoretical biologists on reductionism versus holism has been featured in many publications. Reductionists break systems down to their smallest blocks of interest, trying to recover large-scale phenomena from smallscale mechanisms. Those with a holistic view prefer to deal with aggregated properties of systems first, and then add complexity by considering the inner structure of the system. These different views of theory construction are mirrored in any research field; there are those who would rather build highly simplified models first, adding later new variables in order to increase the realism, while others prefer to start by including every aspect of the system, perhaps eventually discarding many of them as the modelling process proceeds ${ }^{[1-4]}$. The alternative views may thus converge towards similar models but not necessarily. 
For instance, most of the population biology theory arose from very simple differential equations where a single variable represents population density; solutions of these are analyzed mathematically, and potentially compared to abundance estimates from field or lab observations ${ }^{[5,6]}$. Although these models have had a great influence on ecological theory, their aggregated form is particularly difficult to relate to observational biology. As this aggregated view of a population is highly simplified, many extensions have been made to incorporate (1) size, age or physiological structure (leading to coupled systems of differential equations); (2) space (leading to metapopulation models in which a population is broken down into distinct patches, or in the case of continuous space to partial differential equations); (3) discrete generations (leading to difference equation models and matrix models); (4) stochastic effects (leading to birth and death processes and stochastic differential equations). As extensions are added, the models become less analytically tractable and considerably harder to analyze except numerically, and not always with enough confidence and success ${ }^{[7,8]}$. However, the use of such extensions may well be required, to ensure the models are sufficiently realistic to be applicable to specific problems in managing natural systems. Modern theory construction should not be bound by the limits of analytical mathematics. The new field of computational ecology is an attempt to combine more realistic models of ecological systems with the often large data sets available to aid in managing these systems, utilizing techniques of modern computational science to manage the data, visualize model behaviour, and statistically analyze the complex dynamics which arise ${ }^{[9-12]}$. This often involves the use of Geographic Information Systems to provide underlying static or dynamic maps of abiotic and biotic factors, which are of importance in the natural system for specific interest ${ }^{[13]}$. Computational ecology will further intensify as a necessary way to analyze complex problems of natural system management involving the coupling of detailed, spatiallyexplicit ecological models with physical models for abiotic components and the attendant effects on the system of human actions.

One reductionist approach in ecology analyses systems based upon the actions of individuals. Modelling the behaviour of individuals and testing whether this behaviour leads to realistic system-level properties is a natural idea. Individual-based models (IBMs) track the behaviour, growth, reproduction and death of individuals, from which they build up the dynamics of aggregated units such as populations and communities. IBMs were developed sporadically and independently as soon as adequate computers were available. In fact one of the early models that was very influential and contributed significantly to the establishment of IBMs was a forest model ${ }^{[14,15]}$. Giacomini et al. ${ }^{[16]}$ pointed out that traditionally, the dynamic of community assembly was analyzed by means of deterministic models of differential equations, and despite the theoretical advances provided by such models, they are restricted to questions about community-wide features.

Understanding the collective behaviour of communities and populations in terms of individual performance is one of the main goals of theoretical ecology. For instance, the dynamics of tree populations are determined by birth, death, the change in the state of trees (individuals), and the interactions between them, and also by exogenous events such as natural or human disturbances. Individuals (trees) differ with respect to their properties or states, mainly size or age, and they may experience spatially heterogeneous living conditions, such as nutrient supply or physical environmental conditions (intensity of light, humidity, temperature, among others). They may also be affected differentially by random events. These differences among trees lead to a variability in the population which can strongly influence its overall dynamics. IBMs offer an opportunity to link bionomic features to patterns at the community scale, allowing us to understand how trait-based assembly rules can arise by dynamical processes ${ }^{[16]}$.

Many existing forest models can be classified by specific criteria. We can distinguish between empirical (phenomenological) models, which mathematically describe specific behaviour, and theoretical (mechanistic) models with a biological basis, which search for the underlying mechanisms driving already observed phenomena. We can also distinguish between primary, secondary and tertiary models, by examining their treatment of the effects of external factors and constraints on the tree population under study.

Over the last decades significant IBMs have been widely used in mathematical biology ${ }^{[15,17,18]}$. IBMs are used to simulate, study and analyse biotic populations. The use of spatially explicit IBMs has extended in ecology. The ability and 
computational capability in tracking single individual trees makes this modelling methodology valuable in forest studies. At the same time, the treatment of the space has a very important role in the dynamics of tree populations ${ }^{[19]}$. Spatially explicit IBMs are bottom-up approaches to tree communities or forests that build links between the description of the trees at a unit level and macroscopic observations at a community level. Berec's study ${ }^{[20]}$ was the first attempt to collect and synthesize detailed information about four different frameworks in which spatially explicit IBMs could be defined.

Classic mathematical models used in forest dynamics studies usually describe the population of trees through a top-down approach (population-based models). They deal with continuous equations that are applied to the whole population (or to fractions of the population in more structured models) and reflect essential tree mean (or median) properties. The variables taken into account by these kinds of models represent macroscopic quantities and averaged (or typical) individual attributes. The input parameters stand for measurements performed on the whole collective system. Individual activity is implicitly inferred from the overall observed behaviour of the system.

An IBM is based on a population description at the individual level, approach called Lagrangian, as opposed to the population-level framework, called Eulerian (which takes an average of the individual features). A significant number of published forest models containing IBM or PDE strategies to deal with the dynamics of tree populations can facilitate the presentation of features and properties of these two methodologies and illustrate the ideas presented in this study. For example, the articles Kohyama ${ }^{[21-23]}$, Kohyama \& Shigesada ${ }^{[24]}$, and Kohyama \& Takada ${ }^{[25]}$ are particular cases of the use of PDEs. On the other hand, for instance, SORTIE is a well-known published IBM to deal with forests in the north-east of United States and was introduced by Pacala et al. ${ }^{[26]}$ and Pacala \& Deutschman ${ }^{[27]}$, and some tasks were performed in the visualization of the simulation results achieved with this complex model ${ }^{[28]}$. The papers written by Pacala $\&$ Tilman ${ }^{[29]}$, Pacala et al. ${ }^{[30]}$, Bolker \& Pacala ${ }^{[31]}$, Lischke et al. ${ }^{[32]}$, and Moravie \& Robert ${ }^{[33]}$ describe IBMs along the same lines as those used in this study. In recent years, other IBMs have been presented by diverse authors on account of a significant increase in the use of this methodology ${ }^{[34-36]}$.

The general aim of this paper is first the assessment and comparison, followed by the connection of these two approaches to study forest dynamics, namely individual-based and population-based models. If we intend to weigh up the characteristics of these two methodologies, which are currently being used and developed in forest studies, it is convenient to have a wide mental representation and avoid excessively detailed pictures. Therefore, with a general overview of modelling forest dynamics, and in order to evaluate these two types of models, an analysis of these methodologies is carried out following the ideas of a successful strategic planning method used in other research fields, the SWOT analysis. Moreover, a way to attain a deeper understanding of these methodologies is to investigate the connections between both formulations. For this reason, guidelines for constructing an individual tree based model and specific points of attention in the individual-based modelling cycle are provided in a pedagogic style example to illustrate this framework. With the design of this general forest IBM, the procedure to obtain its corresponding Partial Differential Equations (PDEs), the equivalent continuous population-based model or the PDE counterpart, is described in detail. The resulting PDEs are equivalent to this IBM in a way that needs to be clarified and discussed throughout the paper. Because each model is built in response to a particular question and with different purposes, understanding the relative capabilities and possible links between these two approaches is interesting for system modellers and simulators.

The paper is organized as follows. Section 2 includes the SWOT analysis of the two modelling strategies: IBMs and population-based models. Section 3 contains some biological information on the evolutions of tree populations to be incorporated in whatever model we wish to build up, IBM or PDE. Section 4 includes a description and formulation of a general IBM for a tree population to be used in the following section, which builds up its corresponding continuous version. In Section 5 the basic ideas to develop a set of PDEs specifically applied to the IBM prepared are presented. Section 6 mentions some forest models, IBMs and PDEs, to consider and illustrate the ideas of the preceding sections. 


\section{A SWOT analysis for discrete individual-based models versus continuous population-based models in the study of tree populations}

In general, SWOT analysis involves specifying the objective of a project and identifying the internal and external factors, both favourable and unfavourable, to achieving that objective. The purpose of any SWOT analysis is to assess these key factors. That is, it is a strategic planning method used to evaluate the Strengths (S), Weaknesses (W), Opportunities (O) and Threats $(\mathrm{T})$ involved in a project. Although SWOT analysis is generally employed in business and resource management, it can also be used in a knowledge-based non-profit context ${ }^{[37]}$. The objective is the construction of a model to study forest dynamics. SWOT analysis groups key pieces of information into two main categories: i) internal factors, the strengths and weaknesses internal to the model and, ii) external factors, the opportunities and threats presented by the external background. The analysed features of a model can be classified into "Strength" and "Weaknesses". "Opportunities" cover the current and potential applications of each method to specific real systems, and also account for those factors aiding their further development, and "Threats" describe the risks and limitations of each method. The SWOT analysis is often used in academia to highlight these positives and negative factors, being particularly helpful in identifying expansion areas. The adaptation of this analysis method to assess modelling strategies has already been used. For instance, Ferrer and co-authors ${ }^{[38]}$ applied this analysis to evaluate the use of individual and population based models in the field of predictive food microbiology. SWOT analysis is not just one method of categorization; but may be useful to persuade modellers to think about what is actually important in achieving objectives. The internal factors may be viewed as strengths or weaknesses depending upon their impact on model objectives.

To deal with the connection between IBMs and population-based models to study forest dynamics it is compulsory to recognize and distinguish their features. SWOT analysis of these two different approaches will be helpful for an overall discussion, after the passage from discrete to the continuous formulation, in order to identify what is being lost and/or gained. Tables 1 and 2 show the characteristics of both approaches and a tentative classification of their strengths, weaknesses, opportunities and threats in a forest modelling context. These tables should be considered as 'dynamic'; they try to summarize our analysis, but it is not possible to circumvent that some of the reflected facts depend on the specific forest application under study and may vary in the near future with the progress of both approaches. On the other hand, the SWOT analysis can be used as input for the creative generation of possible strategies by asking and answering each of the following four questions: i) How can we use and capitalize on each "Strength"?, ii) How can we improve each "Weakness"?, iii) How can we exploit and benefit from each "Opportunity"?, and iv) How can we mitigate each "Threat"? They are not easy questions to answer; nevertheless, the answers depend on the specific purposes and applications that drive the development of a model.

Some researchers believe that IBMs are characterized by an intuitive design and appearance and are relatively easy to implement in a computer. Many scientists use them to avoid PDEs, mathematical formulations requiring an exact analytical solution or an approximate numerical solution, because they consider the computer simulations more straightforward. In contrast, others think in another way, only giving credibility to a PDE system and its numerical resolution (or its analytical solution, which exists in rare cases). These latter researchers avoid the discrete visualization of the tree community that a forest modeller has when using an IBM. On the other hand, IBMs usually include randomness, because knowledge of phenomena they model usually includes some uncertainty. The stochasticity within IBMs makes them yield different outcomes in different executions of the simulation. Individual based, stochastic models have the potential to realistically describe population dynamics. However, they are not mathematically transparent ${ }^{[32]}$. This event can leave scientists insecure over the results and accuracy of the model. Alternatively, IBMs incorporate more biological complexities for tree behaviours and their interactions than classical and continuous state variable models. In some cases the IBM approach can be less general, applied to more specific situations than those used by classical models. The 
apparent simplicity of some IBMs is compensated for by their greater level of biological details when the species to be simulated are well known. Then, changing model parameters is essentially changing environmental or individual features.

Table 1. List or scheme of "Strengths" or favourable internal factors, "Weaknesses" or unfavourable internal factors, "Opportunities" or favourable external factors, and "Threats" or unfavourable external factors for discrete IBMs to be used in a forest study

\begin{tabular}{|c|c|c|}
\hline Bottom-up approach & HELPFUL & HARMFUL \\
\hline INTERNAL ORIGIN & $\begin{array}{l}\text { STRENGTHS } \\
\text { - A relatively simple structure easy to } \\
\text { - } \quad \text { Explerstand for non-mathematicians } \\
\text { response to the spatial and temporal } \\
\text { variability of individual trees } \\
\text { Biological basis of the parameters defined } \\
\text { at an tree level } \\
\text { Holistic knowledge of the system: } \\
\text { assessment of how collective behaviour } \\
\text { arises from the assumptions stated at tree } \\
\text { level } \\
\text { Capacity to study separately the different } \\
\text { tree factors may cause particular forest } \\
\text { behaviour } \\
\text { Can easily deal with small number of trees } \\
\text { that can have an important effect over the } \\
\text { overall community }\end{array}$ & $\begin{array}{l}\text { WEAKNESSES } \\
\text { - } \quad \text { Lack of standard methodologies to detailed guide } \\
\text { model building and to assess its reliability } \\
\text { Usually slow to implement and often require high } \\
\text { - } \quad \text { Habitually excessively data-hungry } \\
\text { - } \quad \text { Some individual tree parameters difficult to } \\
\text { measure with current experimental } \\
\text { methodologies } \\
\text { Different and big temporal and spatial scales } \\
\text { covered with difficulty } \\
\text { Uncertainty of such single tree-based models at } \\
\text { the stand level is hardly assessable (the error } \\
\text { propagation from the tree to the stand level is } \\
\text { intricate) } \\
\text { Not amenable to analytical methods such as } \\
\text { stability analyses or closed-form solutions }\end{array}$ \\
\hline EXTERNAL ORIGIN & $\begin{array}{l}\text { OPPORTUNITIES } \\
\text { - The way to track the connection between a } \\
\text { population-level defined law and the } \\
\text { biological basis supporting it } \\
\text { - An efficient framework for including and } \\
\text { integrating factors operating at different } \\
\text { spatial and time scales } \\
\text { - Structurally realistic can be used as a } \\
\text { virtual laboratory to experiment and } \\
\text { improve the understanding of processes } \\
\text { Chance to guess tree parameters that are } \\
\text { difficult to measure or indirectly calculate } \\
\text { from experimental observations } \\
\text { Appropriate tool to compile the } \\
\text { information found in databases at a tree } \\
\text { level and make it usable for continuous } \\
\text { models } \\
\text { Statistical advances and increasing } \\
\text { computing power enable a better estimation } \\
\text { of the prediction errors }\end{array}$ & $\begin{array}{l}\text { THREATS } \\
\text { - Difficulty in the analysis of IBMs may hinder the } \\
\text { extraction of synthetic understanding } \\
\text { Complex structure and non-standard variables } \\
\text { and parameters may impede their development, } \\
\text { understanding, comparison and communication } \\
\text { Difficulty in assessing error propagation and the } \\
\text { degree of confidence of a simulation result may } \\
\text { cloud the distinction between coincidence and } \\
\text { cause } \\
\text { The validation of an IBM does not ensure that the } \\
\text { proposed mechanism is the real cause of the } \\
\text { behaviour studied } \\
\text { Comparing a tree-based model with others is } \\
\text { difficult, because the context, the approach and } \\
\text { the complexity are specific to each model }\end{array}$ \\
\hline
\end{tabular}


Table 2. List or scheme of "Strengths" or favourable internal factors, "Weaknesses" or unfavourable internal factors, "Opportunities" or favourable external factors, and "Threats" or unfavourable external factors for continuous and population-based models to be used in a forest study.

\begin{tabular}{|c|c|c|}
\hline Top-down approach & HELPFUL & HARMFUL \\
\hline INTERNAL ORIGIN & $\begin{array}{l}\text { STRENGTHS } \\
\text { - Widely employed and accepted formalism } \\
\text { Standardized methodologies in the process } \\
\text { of building, implementing and testing } \\
\text { models } \\
\text { - Easily usable by other researchers when it } \\
\text { is already programmed by experts } \\
\text { numerical researchers } \\
\text { Rapid and effective in the framework of } \\
\text { forest dynamic in certain institutions of } \\
\text { management or conservation }\end{array}$ & $\begin{array}{l}\text { WEAKNESSES } \\
\text { - Sometimes neglect individual variability and } \\
\text { local interactions, or at least, is difficult to } \\
\text { implement and to manoeuvre } \\
\text { - } \quad \text { Need translation to gain insight into trees } \\
\text { - Hinder biological interpretation of the model's } \\
\text { - } \text { parameters } \\
\text { An extra unwarranted parameter may improve } \\
\text { the model's likelihood but not its reliability } \\
\text { Existing information at a tree level that cannot } \\
\text { easily be integrated at the population level }\end{array}$ \\
\hline EXTERNAL ORIGIN & $\begin{array}{l}\text { OPPORTUNITIES } \\
\text { - New models that incorporate existing } \\
\text { - } \text { Mtandardized models may be developed } \\
\text { vegels developed in the framework of } \\
\text { other fields and vice versa } \\
\text { - Easily provide the criteria for monitoring } \\
\text { and analyzing real systems in the } \\
\text { framework of natural resources } \\
\text { Data from databases referring to real } \\
\text { systems are easily incorporated }\end{array}$ & $\begin{array}{l}\text { THREATS } \\
\text { - Choice of system-level assumptions may } \\
\text { eventually lead to misunderstanding of the tree } \\
\text { individual-causes of an observed phenomenon } \\
\text { The difficulty in specifying the scope of validity } \\
\text { of a certain assumption may lead to incorrect use } \\
\text { of a model outside its domain }\end{array}$ \\
\hline
\end{tabular}

\section{Some basic biological features on forest evolution}

Below we present a few ideas about forest evolution that will help put in context the assumptions made in this work and shared by many other models ${ }^{[9]}$. Some of those ideas are explicitly incorporated in the model presented in the next section. In general, the establishment, growth and mortality of individual trees can be modelled and simulated as a function of biotic and abiotic factors. Here we do not consider forest functions that deal with biogeochemical cycling of carbon and nitrogen, or the flow of water through the ecosystem, or competition-interaction with other life forms. On the other hand, the mortality of a large tree produces a gap in the forest, leading to the release of suppressed trees and increased tree recruitment rates, both of which drive forest succession.

Tree diameter at breast height $(\mathrm{DBH})$ is a commonly used variable, with its growth treated as a deterministic process. A tree's height is obtained through an allometric function of DBH. The optimal growth function of a tree depends on the diameter and is basically limited by the influence of available light, the stand basal area considered and the annual degree-day sum (the climatic influence) fundamentally.

Leaf Area Index (LAI) must be known to reproduce light competition because it is a key measurement for understanding energy and materials exchange rates between forest canopies and the atmosphere, and can be related to the tree diameter. Theoretical and empirical linkage between LAI and light interception, among other processes, is well established ${ }^{\text {[39] }}$. This LAI is defined as the ratio of total upper vegetation leaf surface and surface land area where vegetation grows. It is a dimensionless value, typically ranging from 0 for bare ground to 6 (or to 8 ) for a dense forest. From tree height and leaf area at the top of each tree, light extinction within the canopy is predicted using the Beer-Lambert law ${ }^{[39]}$ which allows the 
calculation of the relative light availability for the tree. Its relative growth performance will depend on this value (due to the relationship with photosynthesis) and its shade tolerance level. The paper of Pacala et al. ${ }^{[30]}$ is especially illustrative as a way to model light influence. In this work we are not considering other resources such as water, nutrients or environmental heterogeneity, because we focus here on the relevance of light as a resource for tree growth. Roughly, tall trees cast shadows and reduce the light intensity received by smaller ones, and light intensity increases the speed of growth. Trees grow and develop bigger and thicker cups, casting shadows over their neighbours. If a tree dies, a gap appears in its location, leading to a new race for light. All these phenomena generate feedback and forest dynamics.

\section{A generic I BM to study forest dynamics}

There are several ways to construct IBMs ${ }^{[15]}$. The purpose here is to mention the basics of a spatially explicit forest model. Some type of spatial grid can be set up; otherwise, a continuum treatment of the space is performed ${ }^{[20]}$. There are two basic methods to track time sequences of individual behaviour in these models. One of them has a fixed underlying time step where all individuals are taken into account, considering changes in behaviour or state variables for each of them. If this is done sequentially, care must be taken to randomize the order of acting individuals to avoid bias. Otherwise, event-based models take each individual to determine when next behaviour or state variable change occurs, and ignores that individual until this time is reached. Such event-driven approaches may be more computationally efficient, as not every individual needs to be investigated each time period. Parallelization methods for IBMs have been developed and indicate that basic model assumptions made for sequential versions may need to be modified in order to use parallel algorithms. The assumptions that could be modified concern the ordering of interactions between individuals and parallel implementations may well handle these in a more realistic manner than sequential implementations.

The implementation of the model structure is influenced by the programming language ${ }^{[40]}$. Conventionally, objectoriented software platforms (e.g., Java and $\mathrm{C}++$ ) are chosen to implement IBMs. The object-oriented paradigm's main advantage is that the code resembles the system being modelled. For example, each individual in a population is represented by an object. Other times procedural languages (e.g., C and FORTRAN) are used and are usually chosen for speed. Due to recent advances, object-oriented programming languages can perform equally well as procedural languages.

\subsection{To create a spatial I BM for tree populations}

Key questions in developing an IBM involve setting appropriate spatial and temporal scales, deciding which individuallevel state variables should be included and how to model them, how to formulate growth processes, reproduction, seed establishment, and mortality of the trees. These are not independent issues as setting the scales affects all these aspects of the model. The appropriate spatial scale would depend upon the activity pattern of a tree over the shortest time period of interest, accessibility of spatially-explicit information on spatial components affecting tree behaviour, and availability of information to accurately model the behaviour of the trees.

Mono-species spatial models can be used in sophisticated evaluations of planting, spacing and harvesting schemes in commercial forests. Vital attributes considered at this point are the modes used by a tree in the establishment, persistence at a site, availability of a method for perseverance at different life stages, and longevity of trees. On account of the level of detail needed, these models synthesize great amounts of data that are usually only available for commercial species, so it is difficult to extend these models to mixed species forests. On the contrary, mixed-species spatial models can be developed to deal with theoretical studies and virtual experiments, which help comprehension of their computational behaviour, and some specific applications with real data. These models can explore the consequences of theories and assumptions on the nature of ecological successions based on the attributes of the species involved. Nevertheless, and for a general case of study, the basic IBM chosen and presented in this paper will be a mono-species spatial model. 


\subsection{A case of study: an IBM for a generic forest}

The present model follows some general tendencies of IBMs designed to study community dynamics.

In order to present the IBM developed for this work, the ODD standard protocol, widely accepted for describing this type of modelling, is used ${ }^{[18,41]}$. The acronym ODD stands for the three blocks of elements used: 'Overview', 'Design concepts' and 'Details', which contribute to further unification of the formulation and implementation of IBMs. This protocol combines a general structure for describing IBMs (making a model's description independent of its specific structure and manner of its implementation) and the language of mathematics (separating verbal considerations from a mathematical description of the equations, rules and schedules that constitute the model). The sequence that is used in the presentation of this forest IBM consists of seven elements: Purpose, State variables and scales, Process overview and scheduling ('O: Overview'), General concepts underlying the design ('D: Design concepts'), Initialization, Input, Sub-models ('D: Details').

\subsubsection{Overview}

\section{Purpose}

This model was developed for analysing generic single-species forest dynamics affected by light, environmental setting, and intra-specific competition. The forest consists of several mono-species trees within a square-shaped area.

\section{State variables and scales}

This model has two hierarchical levels: individual trees and simulated area. At the individual level, tree establishment, growth, mortality and competition for light are considered. At the spatial level it defines a homogeneous area with non-limiting abiotic conditions such as water or nutrient availability. An individual tree is described by its stem position in the continuous spatial domain, its age and its DBH (see Figure 1). The size of the simulation area can be arbitrarily attributed, a typical size being 1 hectare. The simulation time step corresponds to one month.

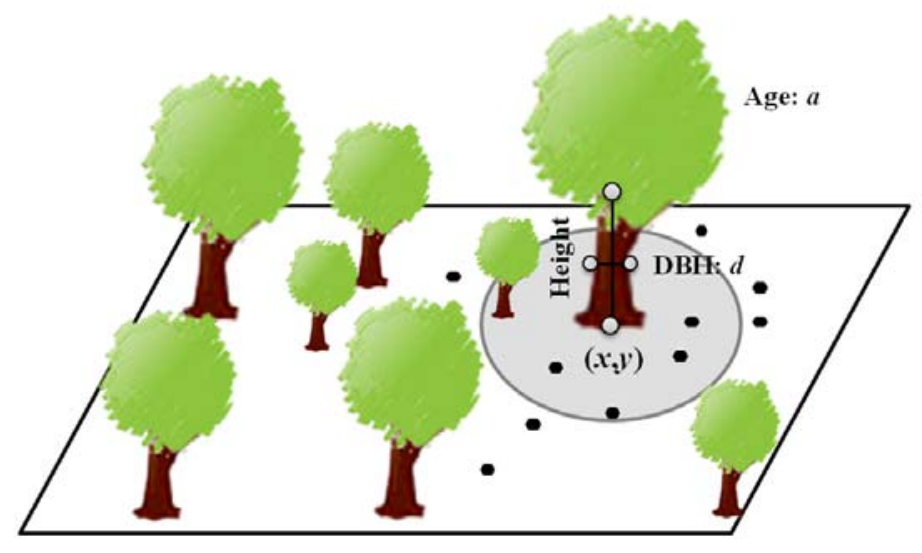

Figure 1. The scheme of the system considered

\section{Process overview and scheduling}

The tree life cycle is described by three biological sub-models operating at monthly time steps. The first sub-model predicts the stem increment of the trees depending on their current stem diameter and light availability. The second simulates tree mortality depending on the age and light absorbed. The third sub-model considers recruitment. Newborn trees settle around the parent tree in a number proportional to the parent's fertility (itself depending on its DBH). The seedling stage is skipped in this sub-model, so that new trees start their life with an initial height $\mathrm{H} 0$ and a stem diameter 
DBH0. The biological sub-models are based on information from specialized literature ${ }^{[42]}$ and other models ${ }^{[9]}$. Each time step, a sequence of processes is operated following the three biological sub-models: establishment, growth and mortality of trees.

\subsubsection{Design concepts}

\section{Emergence}

We explicitly model the growth of each tree depending on surrounding conditions in the search for light, and the resulting mortality. Emergent system dynamics include the size of tree population (with control of dead trees), total biomass evolution, size structure of the forest (DBH classes), distribution of light availability, and spatial location of trees and canopy zones.

\section{Interaction}

Trees compete for light resources. Space is a critical feature of this competition, as long as trees cast shadows over neighbouring trees. This competition is phenomenologically described using the philosophy of "zone of influence models" ${ }^{[32,43]}$. According to this approach, each tree has a circular, size-dependent zone around its stem position where the tree influences its neighbours and is influenced by them. The strength of competition depends on the size and leafiness of the trees. Each tree is assumed to be shadowed by all the taller trees in its neighbourhood (a tree's neighbourhood is seen as a circle of a certain radius around the tree).

\section{Sensing}

Through their leaves, trees perceive light intensity conditions. Individual trees are "informed" about the light conditions at their stem position and the local neighbourhood situation via zone influence overlapping.

\section{Stochasticity}

This model includes stochastic processes. Firstly, newborn trees are established at random positions near the parent tree. Secondly, trees die according to random variables, where the probability distributions of these variables take into account environmental factors and individual features. All these stochastic elements collect all other factors either unknown or not explicitly used in our model. The simulation uses a pseudorandom number generator yielding different outcomes in different executions.

\section{Observation}

This simulator continuously records the state variables such as tree positions, DBHs, and the available light received by each tree. The output files can easily be imported onto a spreadsheet for analysis and visualization. Statistical procedures with stem diameters, tree biomasses, or tree heights (from their allometric relation with DBHs) provide further capabilities to analyse forest development.

\subsubsection{Details}

\section{Initialization}

The initial number of trees is one of the input parameter of the simulation. They are distributed randomly in the domain. Their DBH is also chosen randomly and different probability functions for that variable are provided, namely Gaussian, uniform, and exponential. One of the future goals of IBM simulations is first, to test the population dynamics under different rules and initial distributions, and second, to compare these evolutions with the dynamics shown in nature. Therefore, the initial conditions can be defined without using the real distribution observed in the field data, and it is likely 
that heterogeneities will emerge from those initial conditions, as occurs in nature. So, different initial conditions could be tested in simulation studies.

\section{Input}

Although the dynamics of forests are driven by certain environmental conditions which vary over space and time (seasons, years, rainfall patterns, soil nutrient gradients...), we do not impose any kind of dynamics for these external variables in this study. Only the source of light is considered. The available light above the canopy of the trees is assumed constant through the whole simulation throughout the domain.

\section{Sub-models}

Tree growth

The growth speed of each tree depends on its DBH $d$ and the light $l$ received. How to model this growth speed, particularly its dependence on light intensity, is a key issue because our main goal is to understand the relationship between light and forest height density profile. We chose the following profile:

$$
G(d)=\left\{\begin{array}{lr}
1.2-0.5\left(\frac{d-30}{30}\right)^{4}, & 0 \leq d<0.3 D_{\max } \\
1.2-0.5\left(\frac{d-30}{50}\right)^{4}, & 0.3 D_{\max } \leq d<0.8 D_{\max } \\
0.7-0.7 \frac{d-80}{20}, & 0.8 D_{\max } \leq d \leq D_{\max }
\end{array}\right.
$$

where $D_{\max }$ is the maximum DBH considered. The new diameter computed for the tree is

$$
d_{\text {new }}=d_{\text {old }}+G(d) l
$$

$G$ may be understood as the growth speed under optimal light conditions. The final increment is then reduced by a correction factor 1 considering light availability at the stem position. There is no difference in using height instead of diameter in the previous code, because of the fact that both are equivalent through an allometric relationship.

The computation of light availability is based on asymmetric competition and Beer's law. Roughly speaking, the light availability for each tree can be computed from the basal area above, i.e., the sum of cup areas of trees present in the neighbourhood. Distant trees cast no shadow over the tree considered, hence are not computed, and this results in:

$$
l=0.1+0.9 \exp (- \text { leaff } \cdot L A I)
$$

where leaff is a constant representing the specific leafiness and LAI is

$$
L A I=\sum_{i} 0.0006 d_{i}^{2}
$$

where the summation includes only the trees in the neighbourhood of the focused tree. $L A I$, as defined previously, is the ratio of total upper leaf area divided by land surface area.

\section{Tree mortality}

At each simulation step, the computer must decide whether each tree survives or dies. A tree is assumed to die for one of these reasons, insufficient light intensity $\left(M_{l}\right)$ or great age $\left(M_{a}\right)$. Also, a constant probability of mortality due to other uncontrolled reasons such as events caused by random effects, anthropogenic disturbances, logging, lightning or hurricanes is included. The probability for a certain tree to die between step $t$ and $t+1$ is 


$$
M=0.002+M_{l}+M_{a}-M_{l} M_{a}
$$

where

$$
\begin{gathered}
M_{l}=0.02\left(1-\frac{l-0.1}{0.9}\right) \\
M_{a}=0.02\left(\frac{a}{A_{\max }}\right)^{6}
\end{gathered}
$$

Where $A_{\max }$ is a reference value for the maximum tree age. This treatment keeps the maximum tree age within a reasonable limit.

We assume that $M$ depends only upon the present situation and not on previous history of the tree. However, it would also be reasonable for future versions to include some influence of previous history, because long-term light scarceness can induce the death of a tree ${ }^{[9]}$.

\section{Tree recruitment}

At each simulation step, each tree spreads seeds in its neighbourhood. It is a known fact that only a fraction of the seeds generated by a tree, in the end, turn into young trees as many seeds die before achieving this stage. These early dead seeds have no influence in further evolution of the forest, so they are omitted in this study; only 'effective' seeds are tracked. This does not lead to any loss of information or generality. Each 'effective' seed produces a new tree. Newborn trees start their development (in the simulation) with a height randomly chosen between $I_{D}$ and $I_{D}+0.1\left(D_{\max }-I_{D}\right)$, and age $a=1$; only trees with a DBH $d>0.2 D_{\max }$ are fertile trees.

These sub-models presented are enough to illustrate the aims of this work, but in future research each tree history should be considered, among other individual features or environmental factors. The precise form of the functions $G=G(d)$ and $M=M(l, a)$ is arguable; it is not our intention at the moment to discuss particular functions but to obtain global ideas for these processes. The input values of fertility, leafiness, seed dispersal radius and neighbourhood radius are user-chosen.

\subsection{I mplementation of this forest I BM developed}

A tree is modelled as the following object:

\begin{tabular}{|ll|}
\hline struct tree & \\
float $\mathrm{x} ;$ & $! \mathrm{x}$ position in the 2D terrain \\
float $\mathrm{y} ;$ & $!$ y position in the 2D terrain \\
float diameter; & $!$ tree's diameter \\
float age; & $!$ tree's age \\
float light; & $!$ light received by tree \\
\hline
\end{tabular}

Computationally speaking, a forest is seen as an array of tree structures. Each different tree is assigned an integer number called index (in the array). This index designs a unique tree along the whole simulation and each tree is assigned one sole index. Newborn trees acquire a new index, not previously used, and any index used by a dead tree is not re-used later. The value index is used to access/read/modify/delete data on the tree throughout the simulation. The 'terrain' of the simulation is a rectangle domain_width $\mathrm{x}$ domain_height. Trees will be situated in this $2 \mathrm{D}$ space at coordinates $(x, y)$. IBMs are time-discrete simulations, that is, time is divided in time steps $\Delta t$. As previously remarked, the main processes taken into account are growth, death and recruitment. Space is considered, as trees are influenced by their neighbourhood. With all of this, the forest is a set of trees evolving (growing, dying and being born) on a 2D continuous domain (the space is not discretized). A general IBM for forest succession may be structured in different blocks or parts. 


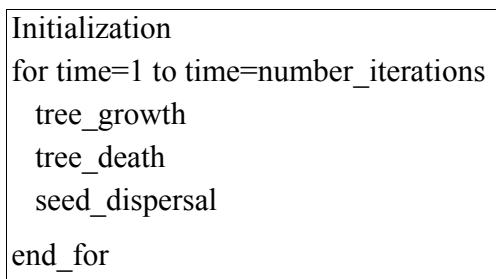

The main blocks of this IBM are tree_growth, tree_death, and seed_dispersal. Of course, these main blocks include subblocks such as computing light intensity, adding trees, deleting trees, aging tress, and others that are more technical (manage computer memory, write data to an output file, compute histograms and statistical data, etc.).

The initialization block is to 'draw' or prepare the initial scenario of the simulation with trees placed in a certain 2D domain, with pre-set ages and heights. A rectangle 2D domain can be considered as the natural scenario of the simulation. At time $t=0$, trees are randomly placed in the domain, following pre-established density distributions.

For instance, a piece of the computer code to calculate the size of the neighbourhood to be used in the growth model must be specified elsewhere in the simulation as follows:

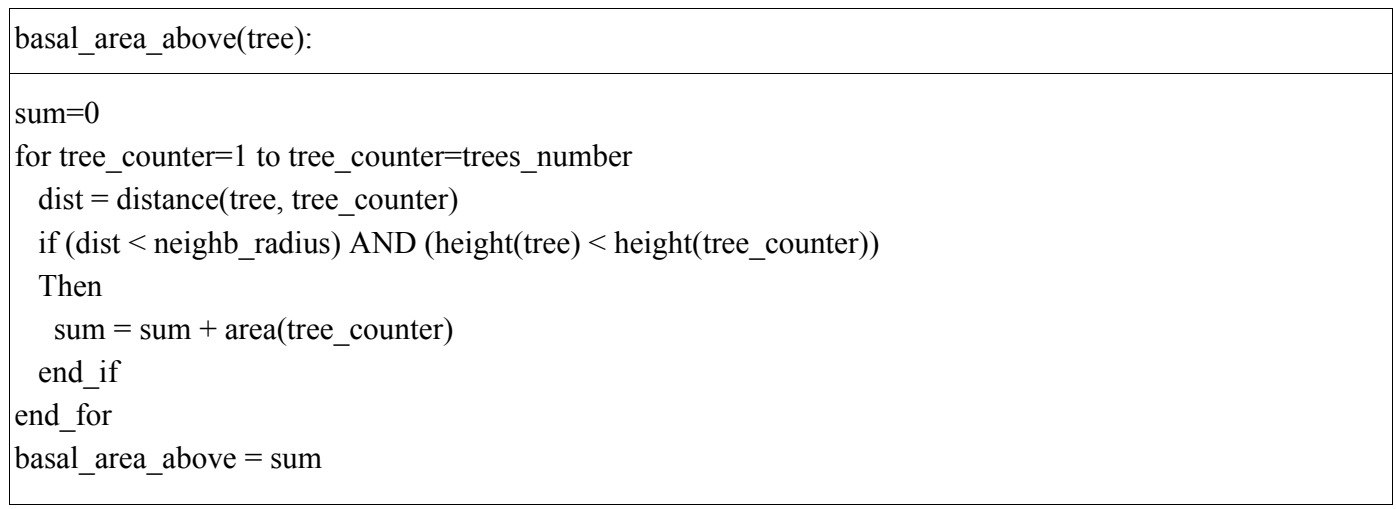

Another code part of the simulator to decide whether a certain tree survives or dies at a specific time step is as follows.

\begin{tabular}{|ll|}
\hline death_decision(tree): & \\
\hline $\mathrm{rn}=$ random_number( 0,1$)$ & $!$ generates a random number between 0 and 1. \\
$\mathrm{M}=$ mortality_rate(tree) & $!$ computes mortality rate \\
if $(\mathrm{rn}<\mathrm{M})$ & \\
$\quad$ delete(tree) & \\
end_if & \\
\hline
\end{tabular}

\section{Obtaining a PDE equivalent to the forest I BM}

\subsection{General considerations}

According to Capasso ${ }^{[44]}$ and Oelschlaeger ${ }^{[45]}$, under certain conditions, there exist Partial Differential Equations (PDEs) approximately equivalent to the IBM. For instance, the way to obtain this equivalent PDE from a particular IBM has already been explained in two different cases: a cancer tumour invasion in a healthy surrounding tissue ${ }^{[46]}$, and a yeast population growth in a liquid medium ${ }^{[47]}$. 
A brief, general overview of the ideas below this passage or connection between PDEs and IBMs is presented in this section, but mathematical details are omitted. For detailed knowledge the interested reader may refer to the previously cited works or specialized literature (s.e. ${ }^{[48-50]}$ ), in addition to some examples of application (e.g. ${ }^{[51]}$ ). Later on we apply these ideas in a study case: a forest succession model, using the IBM explained in the previous section.

The mathematical description of any IBM needs to distinguish between two kinds of processes: continuous and point processes. By continuous processes we mean those being produced at every time $t$ at a certain rate (such as migration, growth and aging), whilst point processes include those induced by qualitative changes (such as birth, death and mutation).

We assume an IBM whose individuals are tagged by integer numbers $k=1, . ., N$. The state of particle $\mathrm{k}$ at simulation step $t$ is represented as the 'generalized position' d-dimensional vector $P^{k}(t)=\left(x_{1}^{k}, x_{2}^{k}, \ldots, x_{d}^{k}\right)$. The components of this vector not only include the spatial position, but also the relevant state variables such as age and diameter (height). Individual $k$ can experience the two types of processes mentioned earlier.

As such, the whole configuration of the system at a certain time $t$ is described by the so-called empirical measure

$$
P_{N}(t)=\frac{1}{N} \sum_{k=1}^{N} \delta_{P^{k}}(t)
$$

Where $\delta_{u}$ represents the Dirac measure at point $u$.

We assume that $\mathrm{PN}(t)$ converges, as $N$ goes to infinite, to a process with density $\rho=\rho\left(x_{1}, x_{2}, \ldots, x_{d}\right)$. Under this assumption and with the requirements set out below the density $\rho$ is the solution of the PDE

$$
\frac{\partial \rho}{\partial t}=-\nabla(\rho v)+b \rho-d \rho
$$

Where $b$ is the recruitment rate, $d$ death rate and $v$ generalized speed under suitable boundary conditions.

It is important to make some remarks about the limitations of this passage from IBMs to PDEs. This passage is not infallible in all cases; it is not expect to be applicable to all-type of IBMs, only to those including motion and demography. For example, IBMs considering communication between individuals or other complex features are untranslatable into PDE terms. Furthermore, as stated in previous paragraphs, the limiting procedure assumes several hypotheses, such as $N \rightarrow \infty$. Obviously, we are not modelling an infinite set of trees. We are not claiming that an exact and precise IBM/PDE equivalence is being presented; we are simply assuming that the IBM is reasonably similar to the limiting PDE formulation obtained. These limitations are relevant and must be kept in mind at all times.

\subsection{Application to the case of study of the forest I BM developed}

The forest model presented takes into account some hypothesis, i.e. the growth process is treated as continuous in time with a rhythm function of light intensity, whilst death and reproduction are punctual phenomena that happen only at certain times. There is no transition between species, and parent trees give birth to daughters of the same species (no mutations).

According to these assumptions, the mathematical description of the IBM is as follows. The state of the $k$-th tree at instant $t$ is the 4-dimensional vector $P^{k}(t)=\left(x^{k}, y^{k}, h^{k}(t), a^{k}(t)\right)$. The pair $\left(x^{k}, y^{k}\right)$ represents the 2-dimensional position on the domain, the component $h^{k}(t)$ stands for tree height, and $a^{k}(t)$ for tree age. The height varies with time and growth rate $g\left(l\left(x^{k}, y^{k}, h^{k}(t)\right) ; a^{k}(t)\right)$, where $g(l ; a)$ stands for growth speed of tree with age a that it is receiving a light intensity $l ; l=l(x, y, h)$ is the light intensity received at position $(x, y)$ and height $h$. Aging evolves at $d a^{k}=d t$ (simply time passing). Birth and death are two point processes, because birth accounts for seed generation at rate $b$, whereas death accounts for trees disappearing at rate $d$. 
As noted in the previous section, all newborn trees have age $a=0$, so the birth term $\mathrm{b}$ will not appear in the evolution equation but in the boundary condition. Building up all these ideas, the reaction-diffusion PDEs system equivalent to the IBM is

$$
\frac{\partial \rho}{\partial t}=-\frac{\partial(g \rho)}{\partial h}-\frac{\partial \rho}{\partial a}+b \rho-d \rho
$$

Where $\rho=\rho(x, y, h, a)$ stands for tree density, with initial conditions $\rho(t=0)=\rho^{*}$ and appropriate boundary conditions (which must be obtained and discussed for the particular domain and forest to be modelled).

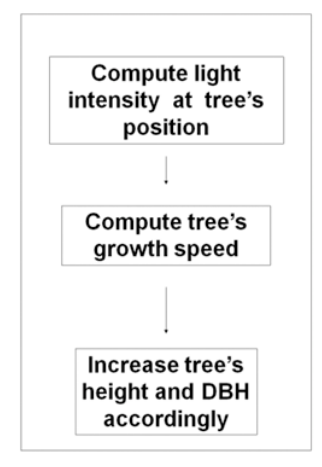

GROWTH

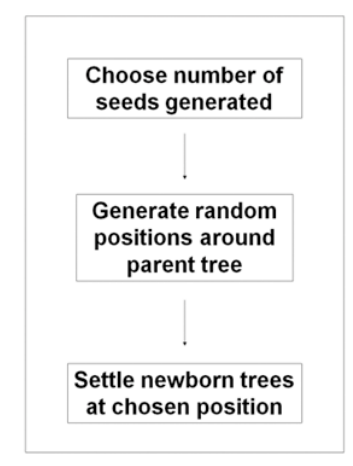

RECRUITMENT

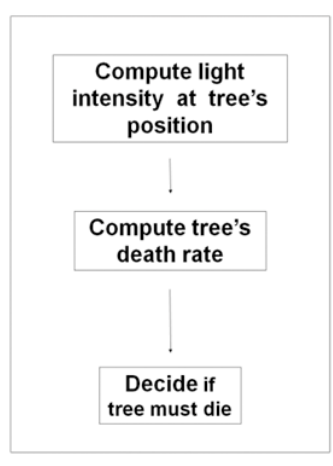

DEATH

Figure 2. Scheme of flow chart of the IBM simulator

\section{Discussion}

In the previous section we have written a PDE inspired by the biological phenomenon under study. We started from a biological description, and then wrote an IBM, and after that obtained the corresponding PDE description. We have attained a classical PDE approach for the same phenomenon. The particular forms of the growth function, shade tolerance, effectiveness of light use, thresholds for tree establishment, mortality rates, among others, are species-specific. So far we have considered a virtual ideal species whose function forms have been chosen in order to be able to reproduce qualitative trends rather than quantitative results. We obviously know that species have very complex and different particularities, but for simplicity it is a good strategy to assume that the features of the species are described well enough by the following four aspects: birth rate function, mortality rate function, growth speed function and tolerance to shade. Light competition and shade tolerance are considered key factors of forest stand structure and dynamics.

Knowing the connection between both levels of description is useful to understand how properties emerge at the PDE level and also to keep the advantages of both: the PDE level for analysis and the IBM level for an intuitive and easy design. The passage for this connection is already well-known for independent particles, but not so well-known when there are interactions between them, as is the case in most biological models, as long as individuals tend to relate between themselves. The question whether the IBM and the PDE obtained are equivalent arises. At first sight, this question may be unessential, because all the steps have a rigorous mathematical nature under certain conditions. However, the fact that the IBM is like a "discretisation" of the differential equations involved in this construction might have introduced some differences between the two models. Another reason may be the different nature of the continuous and discrete approaches; the limit when the number of trees approaches infinity is considered in this connection in order to achieve a continuous description. This passage is intuitively clear, but differences could exist between the finite and infinite cases. The limiting process we used to derive a PDE approach assumes that the number of trees involved in the IBM is sufficiently large, and this can be a reasonable explanation for differences between the outcomes of both approaches. For 
instance, this assumption could not be sufficiently satisfied for the initial stages of the simulation where few trees were handled or after a natural "disaster" where only a small number of trees were alive. The level to which these disparities would arrive could be a motivation for future implementations of these two approaches in order to obtain quantitative results. Another source of dissimilarities could be the stochasticity associated with, or intrinsic to, the rules or updates that take place in the IBM. In the case developed in the previous section, stocasticity has been little used but could be used in a more extended way, for example in the tree growth or in the calculation of light received, but it is not the case with the PDE (stocasticity would not be included in that sense). Many of the features of the IBM are naturally represented by integer values leading to discontinuous jumps along the simulation, and random variables are used in order to introduce variability in the system's evolution and composition. However, the PDE approach cannot reproduce these behaviours accurately, as it yields continuous, deterministic, smooth results and diagrams. The optional addition of a diffusive term in the PDE may be understood as the PDE-scale consequence of individual-scale random events or factors not controlled by the model.

Implementation of the forest IBM designed in a suitable framework to carry out simulations and the numerical resolution of the PDE obtained, would allow us to compare quantitative results. As the IBM includes randomness, the comparison between both approaches should be statistical. This is an interesting point to consider. On one hand, we deal with a deterministic tree density depending on time $\rho(x, y, h, a)$, a numerical solution of the PDE formulated, and on the other hand we can handle a set of trees with their own characteristics from the IBM constructed, and those values must be compared. For every time $t$, both distributions should be similar. To test this similarity we could imagine that the tree density depending on time $\rho(x, y, h, a)$, was taken as the 'theoretical' distribution, while a specific configuration of the IBM was taken as the 'empirical' one. In this line, two previous studies in the area of biology can be cited to illustrate quantitative results in the comparison of IBMs and PDEs, comparisons of the observed macroscopic and microscopic behaviour of an IBM with the corresponding differential equation counterpart of this IBM were shown. The first study is about an application to the upstream movement of elvers ${ }^{[52]}$ and the second is a study on yeast populations ${ }^{[47]}$. These types of works provide a further insight into the possibilities of both modelling tools and their connections.

The numerical resolution of a PDE proves daunting beyond some spatial dimensions, and when studying biological systems, many individual features are usually considered. In the case of the trees further properties or individual features could be added easily, as well as variables related with memory, or previous history of the tree, or individual energy reservoir, which can determine future individual behaviours. While we thought of including a corresponding term in the PDE, it increased the complexity far too much. As roughly each individual feature yields another spatial dimension in the PDE counterpart, the resulting PDE soon becomes intractable. While it is quite simple to add new attributes into the IBM computer code, the same strategy proves unfeasible in the PDE counterpart. The ease of implementation of some individual actions also differs from one approach to the other. While they may be easily included in the IBM, they can correspond to twisted, hard-to-analyse terms in the PDE. It is necessary to use a simplified version of a forest continuous model in order to keep the PDE counterpart reasonable and tractable.

As IBMs are computational models, the forest IBM developed has to be implemented in a computer code and executed in a convenient framework in order to be able to analyse the simulation results or outcomes. It is also true that a forest IBM can carry high computation costs when dealing with many trees which interact and evolve in a changing environment The possibility of having convenient computerized frameworks to deal with IBMs would greatly facilitate this task and may help in the efficient production of the forest simulator. Among other options, such as constructing the computer code with an object-oriented programming language or procedural language, the use of specific platforms prepared to work with IBMs is an alternative. For instance, NetLogo (among other agent or individual-based simulation toolkits) is a good option for this implementation and it is a free tool accessible on the Web (http://ccl.northwestern.edu/netlogo/). The resolution of the PDE with boundary conditions needs numerical recipes and mathematical software when analytical resolution is not possible (in fact, this is only possible with rather simple PDEs). The proper use of these mathematical tools is not trivial. IBMs do not require a profound mathematical knowledge and can be very helpful for non-experts, or those who have previously worked with some kind of discrete modelling, being more accessible and intuitive. However, IBMs are less 
standardized than PDEs as a scientific tool. The game-like appearance of IBM simulations is still considered by some scientists to be a mark of the lack of scientific rigour, something never discussed regarding any PDE approach (although it is true that nowadays, in the biological arena, this feeling is disappearing).

Other kinds of approximations can use different modelling approaches leading to new constructions. The combination of discrete and continuous modelling strategies is a good alternative in some cases, depending on the focus of the problem, combining the strengths of both PDE and IBM models. Lischke et al. ${ }^{[53]}$ present "TreeMig", a spatially explicit, grid-based and spatially linked forest-landscape model. This model is hybrid: within-cell dynamics evolve according to certain height-structured models, whilst inter-cell dynamics are assumed to follow Poisson distributions.

\section{Conclusions}

In this work we have considered the study of the evolution of tree populations in a spatial domain by using two approaches: one, a computational individual-based perspective; the other, a partial differential equations perspective reproducing the same phenomena.

In general, the model type used in the study of forest dynamics or tree populations is based on the problem under consideration, the data available, and the aspiration to develop a malleable model widely adaptable to diverse scenarios. It is desirable that these models could be flexible enough to be used in constantly changing environments, or heterogeneous planted tree genetics, or when external perturbations take place in the forest. One of the purposes of computational researchers, where the generators of IBMs can be included, is to explore modelling techniques in order to achieve a more mechanistic approach, with a better understanding of tree growth and forest yield. IBMs seem to be better prepared to give these answers than PDEs. For instance, the introduction of some of those ideas of changing environments, heterogeneous trees or external perturbations are processes that could not be introduced in the variables and equations of PDEs constructed straightforwardly, in counter-position to the intuitive incorporation of those ideas in the procedures that made up the computer codes of IBMs. We believe IBMs to be valuable, but their limitations must be known and appreciated.

Strengths, weaknesses, opportunities and threats (SWOT) analysis of these two different approaches in the forest context, IBMs and PDEs, has been made to give an overall idea of the possibilities of discrete modelling and continuous modelling dealing with a populations of trees evolving along the time.

The individual-based approach assumes more explicitly the inherent complexity of individuals that the PDEs do. We believe that IBMs can yield better results in specific systems than PDEs. The use of IBMs in forest succession has increased in recent years, perhaps due to the intuitiveness of their modelling and representation. IBMs can now represent the complex interplay between the local environment and each individual tree in the community. Nevertheless, complexity is also the major liability of IBMs as it becomes increasingly difficult to manage this kind of model. Moreover, IBMs are stochastic rule-based models and so, it is convenient to compare and contrast them with other modelling classical techniques such as PDEs.

A bridge between both approaches, IBMs and PDEs, has been developed under certain restrictions or requirements. A sequence of steps and ideas to establish this connection, based on mathematical reasoning, has been exposed in a very general forest model. An equivalent PDE formulation of the forest IBM designed has been obtained as an example of how to proceed. Our passage establishes the relationship between the Lagrangian scale and the Eulerian scale, because an IBM corresponds to the former point of view, and a PDE to the latter.

In comparison, mathematical continuous models are (at least in principle) easy to analyse and easy to communicate (their language is mathematics). Sometimes PDEs can be studied and analysed through standard tools of mathematics, not always due to the use of numerical methods and the convergence of their numerical solutions. But, there is no established 
method to obtain conclusions from an IBM, and modellers obtain results by using their intuition. As (most) IBMs include stochasticity, different runs of the same IBM yield different outcomes; sometimes this randomness does not help in obtaining conclusions from a model.

There is no reason to consider continuous (or more classical) modelling and IBMs as incompatible or exclusive methodologies since they can be complementary to each other, which is convenient for many biosystem modelling projects, specifically related to forests or tree populations. The presentation and discussion of these two modelling methodologies applied to the study of a specific biological system, a population of trees, can contribute to the improvement in the teaching of models and their use for researchers. The fundamental and underlying ideas of the two different approaches, top-down and bottom-up, as well as their relationship, will be helpful for current and future users.

Modelling is perceived as a way of dealing with real life activities. Acquisition of the capacity and ability to autonomously carry out all aspects connected to the modelling process in a specific context is very significant. This contribution is an attempt to facilitate those acquisitions, or at least to transmit the main ideas involved in the two modelling processes chosen here, illustrating them with a specific application in the field of natural science.

\section{Acknowledgements}

Gómez-Mourelo is supported by Ministerio de Educación y Ciencia of Spain (Plan Nacional $\mathrm{I}+\mathrm{D}+\mathrm{i}$ ) through grant MTM2008-06462-C02-01, and Ginovart through Grant MICINN, CGL2010-20160.

\section{References}

[1] Levin S. A., Grenfell B., Hastings A., Perelson A. S., Mathematical and Computational Challenges in Population Biology and Ecosystems Science, Science. 1997; 275: 334-343. PMid:8994023 http://dx.doi.org/10.1126/science.275.5298.334

[2] Peck S. L., Simulation as experiment: a philosophical reassessment for biological modeling, Trends Ecol. Evol. $2004 ; 19: 530-534$. PMid:16701318 http://dx.doi.org/10.1016/j.tree.2004.07.019

[3] Urban D. L., Modeling ecological processes across scales, Ecology. 2005; 86: 1996-2006. http://dx.doi.org/10.1890/04-0918

[4] van Nes E. H., Scheffer M., A strategy to improve the contribution of complex simulation models to ecological theory, Ecol. Model. 2005; 185: 153-164. http://dx.doi.org/10.1016/j.ecolmodel.2004.12.001

[5] Murray J. D., Mathematical Biology, Springer-Verlag, Berlin Heidelberg New York, 1990.

[6] Haefner J. W., Modelling Biological Systems: Principles and Applications, Chapman and Hall, New York, 1996.

[7] Zhanga Y., Hensonb M. A., Kevrekidisc Y. G., Nonlinear model reduction for dynamic analysis of cell population models, Chem. Eng. Sci. 2003; 58: 429-445. http://dx.doi.org/10.1016/S0009-2509(02)00439-6

[8] Antonelli P. L., Rutz S. F., Eco-strain in model forests, Nonlinear Analysis RWA. 2009; 10: 576-588. http://dx.doi.org/10.1016/j.nonrwa.2007.10.013

[9] Bugmann H., A review of forest gap models, Climatic Change. 2001; 51: 259-305. http://dx.doi.org/10.1023/A:1012525626267

[10] Berger U., Hildenbrandt H., Grimm V., Age-related decline in forest production: modelling the effects of growth limitation, neighbourhood competition and self-thinning, J. Ecol. 2004; 92: 846-853. http://dx.doi.org/10.1111/j.0022-0477.2004.00911.x

[11] Phillips P. D., Thompson I. S., Silva J. N. M., van Gardingen P.R., Degen B., Scaling up models of tree competition for tropical forest population genetics simulation, Ecol. Model. 2004; 180: 419-434. http://dx.doi.org/10.1016/j.ecolmodel.2004.04.029

[12] Adame P., Hynynen J., Cañellas I., del Río M., Individual-tree diameter growth model for rebollo oak (Quercus pyrenaica Willd.) coppices, For. Ecol. Manage. 2008; 255: 1011-1022. http://dx.doi.org/10.1016/j.foreco.2007.10.019

[13] Sönmez T., Diameter at breast height-crown diameter prediction models for Picea orientalis, Afr. J. Agric. Res. 2009 ; 4: $215-219$.

[14] Botkin D. B, Janak J. F., Wallis J. R., Some ecological consequences of a computer model of forest growth, J. Ecol. 1972; 60: 849-873. http://dx.doi.org/10.2307/2258570

[15] Grimm V., Railsback S. F., Individual-based modeling and ecology, Princeton and Oxford, Princeton University Press, NJ-USA, 2005.

[16] Giacomini H. C., De Marco JR. P., Petrere JR. M., Exploring community assembly through an individual-based model for trophic interactions, Ecol. Model. 2009; 220: 23-39. http://dx.doi.org/10.1016/j.ecolmodel.2008.09.005

[17] Grimm V., Wyszomirski T., Aikman D., Uchmanski J., Individual-based modelling and ecological theory: synthesis of a workshop, Ecol. Model. 1999; 115: 275-282. http://dx.doi.org/10.1016/S0304-3800(98)00186-0 
[18] Grimm V., Berger U., Bastiansen F., Eliassen S., Ginot V. et al., A standard protocol for describing individual-based and agent-based model, Ecol. Model. 2006; 198: 115-126. http://dx.doi.org/10.1016/j.ecolmodel.2006.04.023

[19] Czárán T., Spatiotemporal Models of Population and Community Dynamics, Chapman \& Hall, London, 1998.

[20] Berec L., Techniques of spatially explicit individual-based models: construction, simulation, and mean-field analysis, Ecol. Model. 2002; 150: 55-81. http://dx.doi.org/10.1016/S0304-3800(01)00463-X

[21] Kohyama T., Simulating Stationary Size Distribution of Trees in Rain Forests, Ann. Bot. 1991; 68: 173-180.

[22] Kohyama T., Size-Structured Multi-Species Model of Rain Forest Trees, Funct. Ecol. 1992; 6: 206-212. http://dx.doi.org/10.2307/2389756

[23] Kohyama T., Size-Structured Tree Populations in Gap-Dynamic Forest-The Forest Architecture Hypothesis for the Stable Coexistence of Species, J. Ecol. 1993; 81: 131-143. http://dx.doi.org/10.2307/2261230

[24] Kohyama T., Shigesada N., A size-distribution-based model of forest dynamics along a latitudinal environmental gradient, Vegetatio. 1995; 121: 117-126. http://dx.doi.org/10.1007/BF00044677

[25] Kohyama T., Takada T., Recruitment rates in forest plots: Gf estimates using growth rates and size distributions, J. Ecol. 1998; 86: 633-639. http://dx.doi.org/10.1046/j.1365-2745.1998.00286.x

[26] Pacala S. W., Canham C. D., Jr. Silander J. A., Forest models defined by field measurements: I. The design of a northeastern forest simulator, Can. J. For. Res. 1993; 23: 1980-1988. http://dx.doi.org/10.1139/x93-249

[27] Pacala S. W., Deutschman D. H., Details that matter: The spatial distribution of individual trees maintains forest ecosystem function, Oikos. 1995; 74: 357-365. http://dx.doi.org/10.2307/3545980

[28] Deutschman D. H., Levin S. A., Devine C., Buttel L. A., Scaling from Trees to Forests: Analysis of a Complex Simulation Model, Science. 1997; 12: 1684.

[29] Pacala S. W., Tilman D., Limiting similarity in mechanistic and spatial models of plant competition in heterogeneous environments, Am. Nat. 1994; 143: 222-257. http://dx.doi.org/10.1086/285602

[30] Pacala S. W., Canham C. D., Saponara J., Silander J. A. Jr., Kobe R. K., Ribbens E., Forest models defined by field measurements: II. Estimation, error analysis and dynamics, Ecol. Monogr. 1996; 66: 1-43. http://dx.doi.org/10.2307/2963479

[31] Bolker B., Pacala S., Using Moment Equations to Understand Stochastically Driven Spatial Pattern Formation in Ecological Systems, Theor. Popul. Biol. 1997; 52: 179-197. PMid:9466960 http://dx.doi.org/10.1006/tpbi.1997.1331

[32] Lischke H., Loeffler T. J., Fischlin A., Aggregation of Individual Trees and Patches in Forest Succession Models: Capturing Variability with Height Structured, Random, Spatial Distributions, Theor. Popul. Biol. 1998; 54: 213-226. PMid:9878601 http://dx.doi.org/10.1006/tpbi.1998.1378

[33] Moravie M. A., Robert A., A model to assess relationships between forest dynamics and spatial structure, J. Vegetation Sci. 2003; 14: 823-834. http://dx.doi.org/10.1111/j.1654-1103.2003.tb02215.x

[34] Wallentin G., Tappeinerb U., Strobla J., Tasser E., Understanding alpine tree line dynamics: an individual-based model, Ecol. Model. 2008; 218: 235-246. http://dx.doi.org/10.1016/j.ecolmodel.2008.07.005

[35] Wernsdörfer H., Rossi V., Cornu G., Oddou-Muratorio S., Gourlet-Fleury S., Impact of uncertainty in tree mortality on the predictions of a tropical forest dynamics model, Ecol. Model. 2008; 218: 290-306. http://dx.doi.org/10.1016/j.ecolmodel.2008.07.017

[36] Fortin M., Bédarda S., DeBloisa J., Meunier S., Assessing and testing prediction uncertainty for single tree-based models: A case study applied to northern hardwood stands in southern Québec, Canada, Ecol. Model. 2009; 220: 2770-2781. http://dx.doi.org/10.1016/j.ecolmodel.2009.06.035

[37] Kong E., The development of strategic management in the non-profit context: Intellectual capital in social service non-profit organizations, Int. J. Manag. Rev. 2008; 10: 281-299. http://dx.doi.org/10.1111/j.1468-2370.2007.00224.x

[38] Ferrer J., Prats C., López D., Vives-Rego J. Mathematical Modelling Methodologies in Predictive Food Microbiology: a SWOT Analysis, Int. J. Food Microbiol. 2009; 134: 2-8. PMid:19217180 http://dx.doi.org/10.1016/j.ijfoodmicro.2009.01.016

[39] Vose J. M., Sullivan N. H., Cliton B. D., Bolstad P. V., Vertical leaf area distribution, light transmittance, and application of the Beer-Lambert Law in four mature hardwood stands in the southern Appalachians, Can. J. For. Res. 1995; 25: 1036-1043. http://dx.doi.org/10.1139/x95-113

[40] Lorek H., Sonnenschein M., Modelling and simulation software to support individual-based ecological modeling, Ecol. Model. 1999; 115: 199-216. http://dx.doi.org/10.1016/S0304-3800(98)00193-8

[41] Grimm V., Berger U., De Angelis D. L., Polhill J. G., Giske J., Railsback S. F. The ODD protocol: A review and first update, Ecol. Model. 2010; 221: 2760-2768. http://dx.doi.org/10.1016/j.ecolmodel.2010.08.019

[42] Kimmins J. P., Forest Ecology, Macmillan Publishing Company, New York, 1987.

[43] Berger U., Hildenbrandt H., A new approach to spatially explicit modelling of forest dynamics: spacing, ageing and neighbourhood competition of mangrove trees, Ecol. Model. 2000; 132: 287-302. http://dx.doi.org/10.1016/S0304-3800(00)00298-2 
[44] Capasso V. Stochastic differential equations and systems of interacting particles. 1st ESMTB summer school lecture notes. 2000; 133-149.

[45] Oelschlaeger K., On the derivation of reaction-diffusion equations as limit dynamics of systems of moderately interacting stochastic many particle processes, Probab. Theor. Relat. Field. 1989; 82: 565-586. http://dx.doi.org/10.1007/BF00341284

[46] Gómez-Mourelo P., Sánchez E., Casasús L., Webb G. F., A fully continuous individual-based model of tumor cell evolution, C. R. Biologies. 2008; 331: 823-836. PMid:18940697 http://dx.doi.org/10.1016/j.crvi.2008.08.010

[47] Gómez-Mourelo P., Ginovart M., The differential equation counterpart of an individual-based model for yeast population growth, Comput. Math. Appl. 2009; 58: 1360-1369. http://dx.doi.org/10.1016/j.camwa.2009.05.024

[48] Liggett T. M., Interacting particle systems, Springer, New York, 1985. http://dx.doi.org/10.1007/978-1-4613-8542-4

[49] Cantrell R. S., Cosner C., Spatial ecology via reaction-diffusion equations, John Wiley and Sons, UK, 2003.

[50] Morale D., Capasso V., Oelschlaeger K., An interacting particle system modelling aggregation behaviour: from individuals to populations, J. Math. Biol. 2005; 50: 49-66. PMid:15692840 http://dx.doi.org/10.1007/s00285-004-0279-1

[51] Stevens A., Derivation of chemotaxis-equations as limit dynamics of moderately interacting stochastic many particle systems, SIAM J. Appl. Math. 2000; 61: 183-212. http://dx.doi.org/10.1137/S0036139998342065

[52] Gómez-Mourelo P., From individual-based models to partial differential equations. An application to the upstream movement of elvers, Ecol. Model. 2005; 188: 93-111. http://dx.doi.org/10.1016/j.ecolmodel.2005.05.014

[53] Lischke H., Zimmermann N. E., Bolliger J., Rickebusch S., Löffler T. J., TreeMig: a forest-landscape model for simulating spatio-temporal patterns from stand to landscape scale, Ecol. Model. 2006; 199: 409-420.

http://dx.doi.org/10.1016/j.ecolmodel.2005.11.046 